\title{
Resúmenes de Trabajos Nacionales
}

\author{
1. ABORTO INDUCIDO EN COLOMBIA, UNA VISION INCOMPLETA \\ Y DESACTUALIZADA DEL PROBLEMA
}

Dr. Alberto Rizo G.

Entre 1960 y el mes de octubre de 1977 , aparecieron en Colombia 72 publicaciones en relación con el aborto inducido.

Del total de artículos publicados en el país, el $36.1 \%$ correspondió a estudios realizados en clínicas u hospitales, utilizando datos proporcionados por las historias clínicas de pacientes ingresadas con diagnóstico de aborto incompleto.

Aproximadamente el $20 \%$, correspondió a encuestas en comunidad a estudios de carácter epidemiológico efectuados para conocer la magnitud de aborto provocado en la comunidad.

El $14 \%$ de los artículos publicados, estudió el aborto desde el punto de vista legal. El restante $30 \%$, de las publicaciones correspondió a artículos varios: anticoncepción, infección, aspectos psico-sociales del aborto y algunos otros pocos sin clasificar.
La gran mayoría de los trabajos sobre aborto, realizados en Colombia en los 17 años que se revisaron, son de tipo descriptivo (recuentos de casos, estudio de registros estadísticos y unas pocas encuestas en la población). Ninguno de los estudios es de tipo analítico (estudio de conglomerados o de casos y controles). No se encuentra ninguno de tipo experimental (simulación o experimentos controlados) .

Se anota que la mayoría de los estudios sobre aborto en Colombia que pudieran proporcionar un estimado acerca de la verdadera magnitud del fenómeno o de sus características, tienen entre 10 a 15 años de efectuados.

Se hace un Ilamado al especialista Gineco-Obstetra colombiano para que se informe acerca de las limitaciones y posibilidades de la metodología para el estudio del aborto que se produce fuera del árnbito de las instituciones de atención médica. 


\title{
2. ANALISIS GRAFICO DEL TRABAJO DE PARTO
}

\author{
Dres.: Carlos J. Pilonieta, Luis A. Lara y Reinaldo Heredia
}

Desde los estudios de Friedman, la evolución del trábajo de parto se ha definido por medios estadísticos y gráficas basados en la dilatación progresiva del cervix. Es así como, utilizando una gráfica en la cual las ordenadas registren la dilatación cervical en centímetros comparado con el tiempo en horas, permite una interpretación clínica del trabajo y un diagnóstico temprano de las alteraciones del mismo.

Siguiendo este concepto se analiza el trabajo de parto de 122 pacientes primigestantes, usuarias del Hospital Mi- litar Central, controladas mediante el sistema de graficación del trabajo. Se compara con un grupo similar de pacientes controladas durante su trabajo de parto mediante el sistema tradicional de línea horizontal. Se valoran las ventajas del sistema gráfico y se demuestran sus beneficios al contribuir a una mejor morbimortalidad fetal.

Se enfatiza en la necesidad de una reestructuración de la historia clínica como método de control activo del trabajo de parto para centros docentes.

\section{LAS PRUEBAS falsas REACtIVAS EN EL MANEJO DE LA SIFILIS. REACCIONES FALSAS EN EL EMBARAZO}

\author{
Dres.: Miguel Guzmán Urrego, Roberto Vergara Támara, Mario Salazar Niño, \\ Rosa Stella Vargas, Alvaro Aguilera y Dora Ramírez de Uribe.
}

En el manejo serológico de la Sífilis, se presenta con frecuencia la situación de enfrentar problemas en que una reacción VDRL es reactiva sin que exista Sífilis (1). Corresponden a las reacciones falsas positivas (2).

Conviene, por lo tanto, conocer y estudiar aquellas circunstancias en las cuales esta reacción puede ocurrir. Una de tales es el embarazo (3). Aunque algunos autores han informado este hecho, los estudios realizados no muestran consistencia en sus resultados, (4), y además han sido realizados sobre universos dispersos (5).

El presente trabajo se realizó como parte de un proyecto destinado a clarificar las reacciones falsas positivas en un grupo de situaciones que existen en nuestro medio. Los objetivos propuestos para él fueron los siguientes:
10. Determinar la frecuencia real de las reacciones falsas positivas para Sífilis en el embarazo.

2o. Determinar qué factores, además del embarazó, pueden estar condicionando la falsa reactividad.

3o. Determinar si existe o no una relación entre la aparición de la falsa reactividad y un perforado particular del embarazo.

40. Determinar, para aquellas falsas reactivas, el tiempo de negativización después del parto.

5o. Determinar la frecuencia real de la Sífilis en la población estudiada.

Se presenta un análisis de las reacciones falsas positivas en el embarazo: Los resultados sobre un universo res- 
tringido de 13.477 gestantes, analizadas las muestras obtenidas de 4.369 , son como sigue: 64 muestras VDRL reactivas y FTA-ABS no reactivas, Io cual da $1.47 \%$ de falsas reactivas; 25 reacciones VDRL reactivas, FTA-ABS reactivas para $0.57 \%$ de Sífilis. Igualmente se encontró que no existe una distribución específica por grupos de edad. Las reacciones falsas reactivas están más asociadas con el primer embarazo. No existe relación entre falsa reactividad y antecedentes patológicos. Las reacciones falsas positivas lo son a títulos muy bajos de $0-2$ diluciones. Se encontró una marcada relación entre ocurrencia de falsas reactivas y el grupo sanguíneo Rh-A.
El seguimiento post-parto mostró que las reacciones falsas positivas se negativizan a los seis meses.

Se encontró que la falsa reactividad no está asociada con un período especial del embarazo ya que aparece desde los primeros meses.

\title{
4. ANTICUERPOS ANTIESPERMA EN LA CLINICA DE ESTERILIDAD
}

\author{
Dres.: William Onatra, Miguel Guzmán, Jairo Barragán y Maribel Ayala
}

\section{Objetivos}

Es bien conocido que dentro de la población de parejas estériles un $15.40 \%$ de las mujeres, presentan títulos de anticuerpos, antiespermas circulantes con un título mayor de 1:16.

Es interés de los autores conocer cuál es la incidencia y qué papel desempeña como causa de esterilidad en nuestro medio.

\section{Materiales y Métodos}

Se practicaron estudios inmunológicos cruzando semen homólogo y suero de uno o ambos miembros de la pareja, para determinación de anticuerpos (según la técnica de Kibrick macroaglutinación) en 60 parejas que consultaron por esterilidad. Como control se tomaron 30 pacientes con embarazos normales entre 28 y 34 semanas, cuyo sue- ro se cruzó con semen normal heterólogo.

\section{Resultados}

Entre las parejas estériles el promedio de edad fue de 28.9 años para las mujeres y de 31.4 años para los hombres. Títulos positivos de anticuerpos antiesperma se encontraron en $44.22 \%$ de la muestra, correspondiendo el $32.69 \%$ a casos con esterilidad primaria.

En el grupo control con un promedio de edad de 24.6 años, el $7.69 \%$ presentaron anticuerpos.

Se correlacionaron los títulos positivos con otras causas de esterilidad y con el recuento espermático; encontrándose como causa única en el $3.84 \%$, asociada especialmente a obstrucción tubárica en el $53.84 \%$ y a recuento espermático normal $(46.6 \%)$. 


\section{BROMOERGOCRIPTINA EN PACIENTES CON HIPERPROLACTINEMIA}

Dres.: Fabio Sánchez Escobar, Jairo García, Alberto Abada y William Mejia

Tradicionalmente se ha utilizado la Bromoergocriptina a la dosis de 7.5 $\mathrm{mg} / \mathrm{día}$. Se evaluó la dosis antiprolactinemia de otros esquemas terapéuticos.

10. $1.25 \mathrm{mg} /$ día $\times 8$ días.

2o. $2.50 \mathrm{mg} / \mathrm{día}$.

3o. $3.75 \mathrm{mg} /$ día.

4o. $5.00 \mathrm{mg} / \mathrm{día}$.

5o. $6.25 \mathrm{mg} / \mathrm{día}$.

6o. $7.50 \mathrm{mg} / \mathrm{día}$.

En dosis fraccionadas cada 6 a 8 horas y al final del esquema utilizado se determina la prolactina.

De acuerdo a los resultados se puede apreciar:

10. Normalización en algunas pacientes de la prolactina con dosis tan pequeñas como $1.25 \mathrm{mg} /$ día.
20. En otros pacientes, resistencia a la acción de la bromoergocriptina, con dosis tan elevadas como $10 \mathrm{mg} /$ día.

3o. Caída de la prolactina a niveles sub-óptimos en pacientes con PRL normal.

De lo anterior se concluye:

10. La dosis terapéutica de la bromoergocriptina, es variable en cada paciente dependiendo de la respuesta farmacológica.

20. La bromoergocriptina utilizada en dosis elevadas, puede llevar a niveles sub-óptimos y desencadenar un cuerpo lúteo insuficiente.

3o. La bromoergocriptina utilizada en personas normoprolatinémicas, desencadena niveles sub-óptimos y por lo tanto cuerpo lúteo insuficiente. 


\title{
PROXIMOS CONGRESOS
}

\section{SIMPOSIO MUNDIAL DE GINECOLOGIA PEDIATRICA $Y$ DE LA ADOLESCENCIA}

2 a 5 de diciembre de 1981. Punta del Este. Uruguay.

\section{Temas}

Efectos de la malnutrición en los problemas obstétricos y ginecológicos de la niña, Oncología ginecológica en la niña y la adolescente, Fertilidad en la adolescente.

Métodos de atención a la niña y la adoleccente, Neuroendocrinología de la niña y la adolescente, Medicina fetal.

\section{Cursos}

Malformaciones congénitas, Patología ginecológica de la niña y la adolescente en la práctica diaria.

\section{Secretaría e informes}

\author{
Congresos y Convenciones \\ Juncal 1305 oficina 1202 Montevideo, Uruguay
}

\section{Coordinador para Colombia}

Dr. Alberto Duarte Contreras

Apartado Aéreo 849. Cúcuta.

\section{CURSO DE POSTGRADO}

Actualización en el diagnóstico y manejo de la Infertilidad. Endocrinología Reproductiva y Anticoncepción.

Entre el 26 y el 28 de Marzo de 1981, se desarrollará en el departamento de Obstetricia y Ginecología, Universidad de Texas San Ántonio, USA, bajo la dirección del Dr. Ricardo H Asch y la colaboración de los Dres. Oscar Kletzy, Nilsa Patriti de Laborde, Sergio Stone y Aquiles Sobrero.

El Curso está diseñado para médicos residentes, médicos en práctica de medicina general y ginecología. El contenido del curso está diseñado para proveer información práctica en el maneio clínico en endocrinología ginecológica, esterilidad del hombre y la mujer y para actualizar la información en anticoncepción femenina y masculina.

El programa es válido para 18 horas de crédito de la Academy of Family Physicians y el American College of Obstetrics and Gynecology (USA).

El Curso está limitado a los primeros 200 aplicantes. Para obtener información comunicarse con el Dr. Ricardo H. Asch, Director de Investigación Clínica, Dept. OB-GYN, 7703 Floyd Curl Dr., San Antonio, Texas, 78284, USA. 


\title{
ACADEMIA NACIONAL DE MEDICINA
}

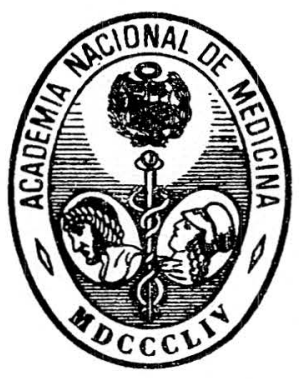

Presidente Honorario: DR. JORGE AVENDAÑO HUBNER Presidente: DR. CARLOS BUSTAMANTE RUIZ

\author{
CONGRESO MEDICO \\ "CARLOS ENRIQUE PAZ SOLDAN" \\ Noviembre 30 - Diciembre 3, de 1980
}

TEMA OFICIAL: "TABAQUISMO"

(Año de la "Lucha contra el Tabaquismo" O. M. S.) Con auspicio oficial y del Instituto Hipólito Unanue intervienen las siguientes Sociedades Científicas:

-- Cardiología

- Angiología

- Gastroenterología
- Psiquiatría, Neurología y Neurocirugía

- Cancerología

- Tisiología y Enf. Aparato Respiratorio.

Cursillos paralelos sobre Aspectos Diagnósticos y Terapéuticos en: Aparato Cardiovascular y Aparato Respiratorio.

\section{COMITE ORGANIZADOR}

Presidente:

DR. JORGE VOTO BERNALES

Vicepresidentes:

DR. CESAR DELGADO CORNEJO

DR. JUAN PHILIPPS VERTIZ

Coordinación General:

DR. ENRIQUE FERNANDEZ ENRIQUEZ

DR. VITALIANO MANRIQUE VALDIVIA

DR. GERMAN GARRIDO KLINGE

DR. CESAR ZAPATA VARGAS

Secretaría General:

DR. CARLOS LANFRANCO LA HOZ
DR. MANUEL SALCEDO FERNANDINI

DR. VICENTE ZAPATA ORTIZ

DR. ALBERTO CAZORLA TALLERI

\section{Economía:}

DR. RICARDO H. CORNEJO

DR. LUIS PINILLOS GOICOCHEA

DR. JORGE DE LA FLOR VALLE

DR. TEOBALDO PINZAS GALLARDO

Relaciones Públicas, Publicaciones y Prensa DR. BALTAZAR CARAVEDO

DR. ERNESTO BANCALARI

DR. ARTURO JIMENEZ BORJA

DR. FAUSTO GARMENDIA LORENA

\section{CORRESPONDENCIA E INSCRIPCIONES:}

Secretario General del Comité Organizador: Dr. Carlos Lanfranco La Hoz

Camaná 773 - Lima - Teléfono 289261

Local de la Academia Nacional de Medicina 